\title{
Osteonecrose do cuneiforme intermédio: Relato de caso*
}

\section{Osteonecrosis of the Intermediate Cuneiform: A Case Report}

\author{
Gustavo Araujo Nunes ${ }^{1,2,3}$ Matheus Levy Almeida Taveira de Souza ${ }^{4(-)}$ Bruno Maciel Braga ${ }^{5}$ \\ Mateus Martins Marcatti ${ }^{1,2}$ Fabrício Melo Bertolini ${ }^{2}$ Otaviano de Oliveira Junior ${ }^{1,2}{ }^{-0}$
}

${ }^{1}$ Departamento de Ortopedia, Instituto Orizonti, Belo Horizonte, MG, Brasil

${ }^{2}$ Grupo de Cirurgia do Pé e Tornozelo, Hospital Universitário Ciências Médicas (FCMMG), Belo Horizonte, MG, Brasil

${ }^{3}$ Departamento de Ortopedia, Hospital da Baleia, Belo Horizonte,

MG, Brasil

${ }^{4}$ Departamento de Ortopedia, Hospital Mater Dei, Belo Horizonte,

MG, Brasil

${ }^{5}$ Departamento de Ortopedia, Hospital São José, Conselheiro

Lafaiete, MG, Brasil

Rev Bras Ortop 2021;56(3):394-398.
Endereço para correspondência Gustavo Araujo Nunes, MD, Rua Prof. Otavio Coelho de Magalhaes, 111, Mangabeiras, Belo Horizonte, MG 30210-300, Brasil (e-mail: gustavoanunes@hotmail.com).

\section{Resumo \\ Palavras-chave \\ - osteonecrose \\ - ossos cuneiformes \\ - adulto}

A osteonecrose é uma doença que raramente afeta os ossos do pé. Quando presente, ela é mais comum no tálus e no navicular. Casos de osteonecrose do cuneiforme intermédio são extremamente raros, e após uma revisão bibliográfica minuciosa encontramos apenas cinco relatos na literatura, sendo todos eles em pacientes pediátricos. A seguir, apresentamos o caso de um paciente adulto com osteonecrose do cuneiforme intermédio resistente ao tratamento conservador. Portanto, propusemos uma abordagem cirúrgica com bom resultado. Nível de Evidência V; Estudos Terapêuticos; Opinião de Especialista.

Osteonecrosis is a disease that rarely affects the bones of the foot. When present, it is more common in the talus and in the navicular. Cases of osteonecrosis of the intermediate cuneiform are extremely rare, and after a thorough bibliographic review, we found only five reports in the literature, all of them in pediatric patients. Below, we present the case of an adult patient with osteonecrosis of the intermediate cuneiform that was resistant to conservative treatment. Thus, we proposed a surgical approach with good results. Level of Evidence V; Therapeutic Studies; Expert Opinion.

\footnotetext{
Trabalho realizado no Hospital Universitário Ciências Médicas (FCMMG), Belo Horizonte, MG, Brasil.
}

recebido

27 de Março de 2020

aceito

01 de Junho de 2020

Publicado on-line

Setembro 25, 2020

\section{Introdução}

A osteonecrose, também conhecida como necrose avascular, é causada pela redução do aporte sanguíneo aos ossos gerando
DOI https://doi.org/ 10.1055/s-0040-1715513. ISSN 0102-3616.

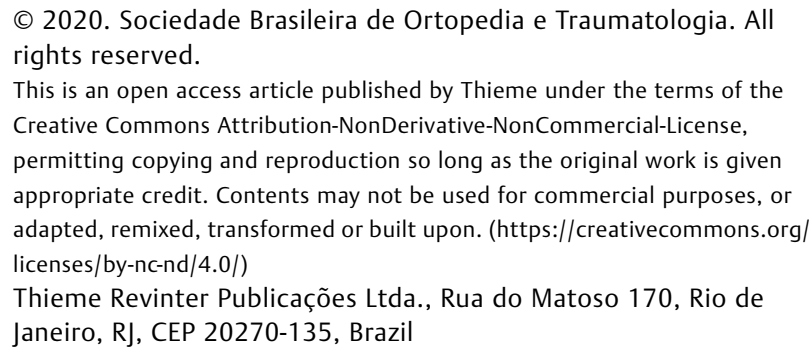




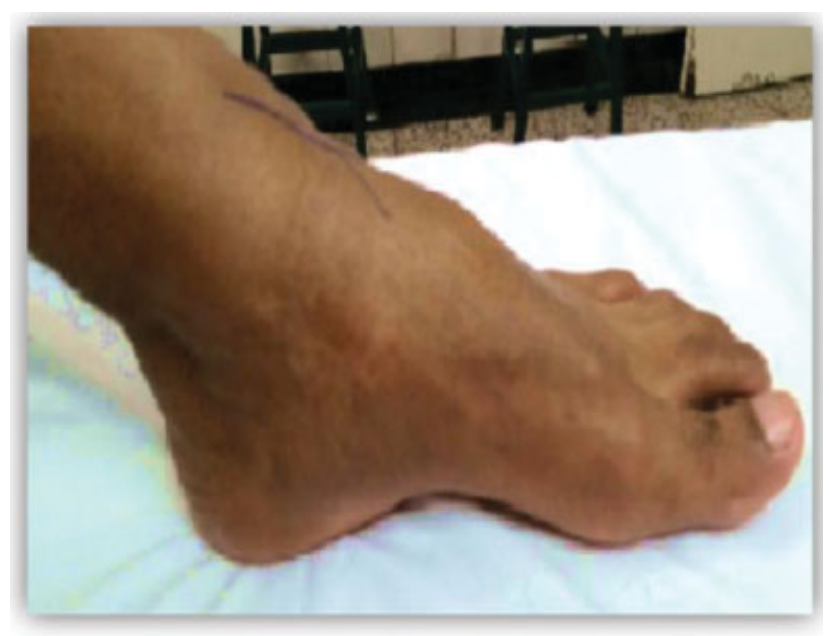

Fig. 1 Fotografia do pé em perfil. Nota-se edema na região dorsal do mediopé associado a um aumento do arco plantar medial. Fonte: Arquivo pessoal do autor.

falha do mecanismo de reposição óssea. Se não for diagnosticada e tratada corretamente pode evoluir com colapso e degeneração articular. ${ }^{1}$ A osteonecrose não é comum nos ossos do pé, e quando ocorre é mais prevalente no tálus e no navicular. ${ }^{1,2} \mathrm{O}$ acometimento do cuneiforme intermédio é extremamente raro, com apenas cinco relatos de casos descritos na literatura, ${ }^{3}$ todos em pacientes pediátricos.

Esta patologia geralmente acomete atletas e ou militares sujeitos a microtraumas de repetição e fraturas por estresse, ou aqueles com doenças reumatológicas em uso crônico de corticosteroide. Outros fatores de risco associados são: radioterapia, quimioterapia, transplante de órgãos e abuso de álcool. $^{2} \mathrm{O}$ tratamento inicial é conservador, com retirada da carga, uso de órteses, e fisioterapia. A intervenção cirúrgica é indicada nos casos em que não há alívio da dor. Existem algumas técnicas relatadas na literatura; porém, devido à raridade e à escassez de estudos dessa patologia no osso cuneiforme, o tratamento ainda permanece controverso e desafiador. ${ }^{1,2}$
O objetivo do presente artigo é relatar um caso raro de osteonecrose do osso cuneiforme intermédio em um paciente adulto tratado através de uma abordagem cirúrgica proposta pelos autores.

\section{Relato do Caso}

O presente trabalho foi submetido ao Comitê de Ética com registro na Plataforma Brasil sob o número do CAAE: 99919318.6.0000.5122.

O paciente DL, do sexo masculino, 24 anos, militar, sem comorbidades, apresentava dor e edema na região dorsal do pé, sem história de trauma, associado à limitação das atividades esportivas, com piora progressiva há aproximadamente 6 meses.

No exame físico, foi observado um pé cavo varo sutil, flexível, que corrigia no primeiro tempo do teste dos blocos de Coleman, associado a um encurtamento do músculo gastrocnêmio demonstrado pelo teste de Silverskiold, além de um edema e dor a palpação no dorso do mediopé. (- Figura 1). Não havia sinais de instabilidade ligamentar e tendinopatias associadas. No exame radiográfico (-Figura $\mathbf{2 A}$ ) notou-se uma linha radiolucente na cortical dorsal do osso cuneiforme, o ângulo formado entre o solo e a borda inferior do calcâneo (pitch do calcâneo) apresentava discreto aumento, com valor de $27^{\circ}$, e o ângulo entre o eixo do tálus e primeiro metatarso (ângulo de Meary) normal. A ressonância magnética (RM) mostrou nas sequências ponderadas em T2 áreas difusas com hipersinal (edema ósseo) associadas a linhas verticais com hipossinal, sugerindo áreas de necrose óssea. (-Figura 2B).

O paciente foi incialmente submetido ao tratamento conservador realizado através da retirada do apoio e uso de órtese até melhora do quadro álgico, seguido de reabilitação fisioterápica e tentativa de retorno gradual às atividades habituais. Não houve evolução satisfatória com o tratamento conservador, e devido a isto, ao tempo de evolução e aos achados no exame de imagem (RM), optou-se pelo tratamento cirúrgico.

A primeira cirurgia foi realizada através do acesso cirúrgico dorsal ao osso cuneiforme intermédio, no qual visualizamos
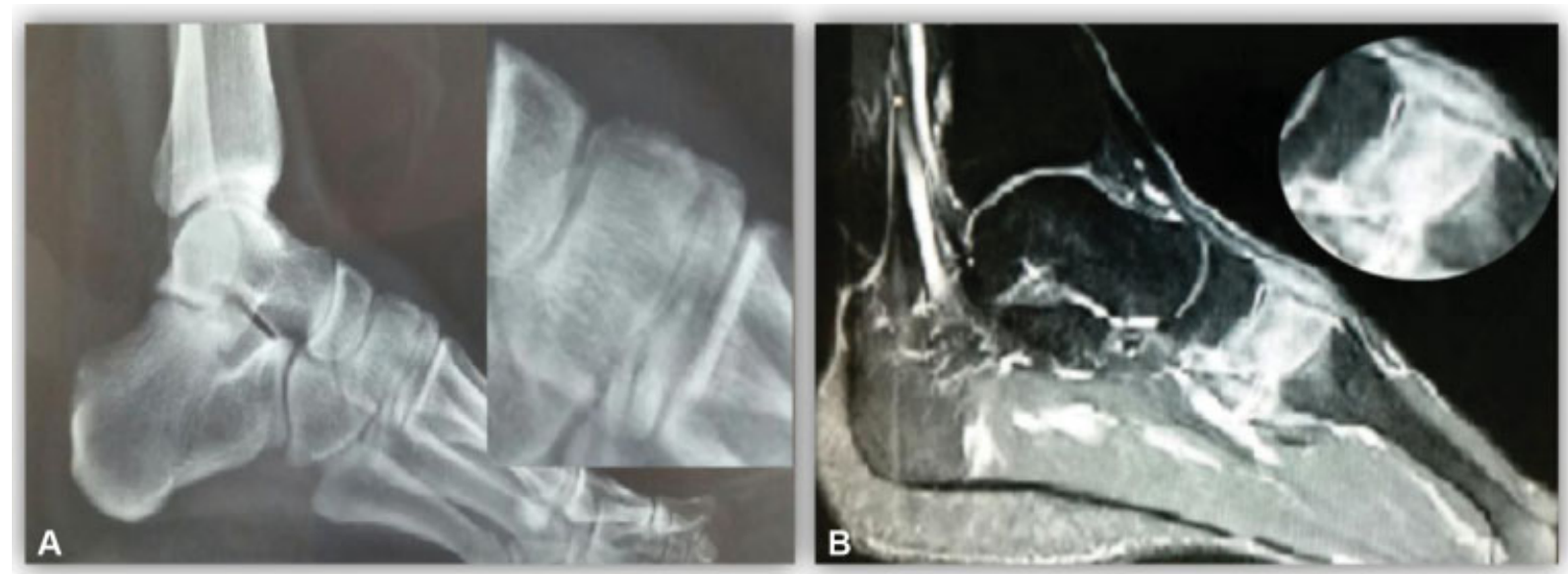

Fig. 2 (A) Exame Radiográfico na incidência perfil. Nota-se linha radiolucente na cortical dorsal do cuneiforme. (B) Ressonância magnética no corte sagital ponderada em T1 demonstrando padrão misto de um hipersinal difuso alternado com áreas de hipossinal. 

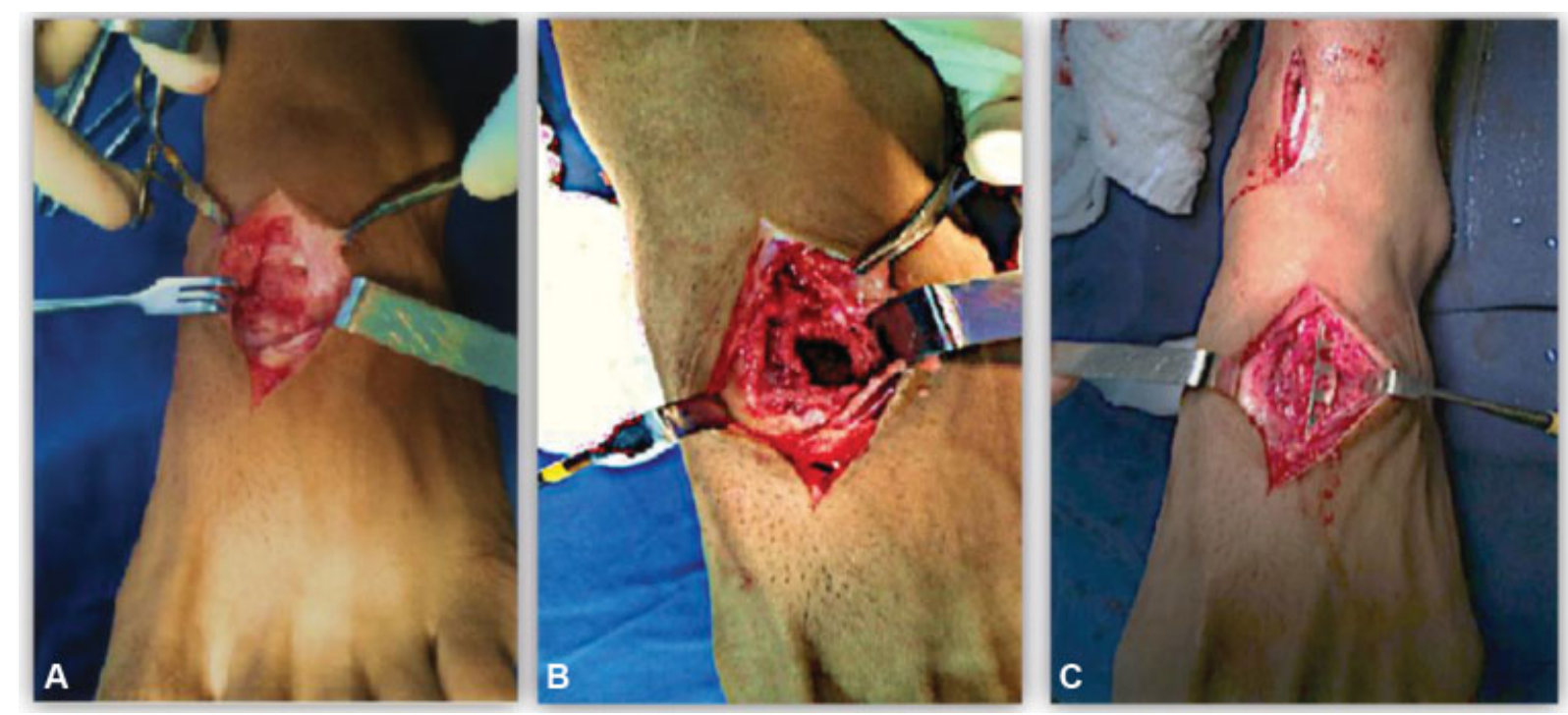

Fig. 3 (A) Acesso cirúrgico dorsal ao cuneiforme intermédio. Observa-se osso necrótico com bordas mal definidas. (B) Janela óssea dorsal seguida de curetagem óssea e ressecção do tecido desvitalizado e necrótico. (C) Preenchimento ósseo com enxerto autólogo fixado com placa ponte. Fonte: Arquivo pessoal do autor.

um osso necrótico com extensa zona de reabsorção, preservando apenas as superfícies articulares. Foram feitos a abertura óssea com janela dorsal, o desbridamento e a curetagem para retirada do tecido necrótico e desvitalizado. Após esta etapa, realizamos o preenchimento da medular do osso cuneiforme intermédio com enxerto ósseo esponjoso autólogo, retirado da tíbia distal. Com o intuito de estabilizar o enxerto e dissipar forças do mediopé, foi posicionada a janela óssea cortical retirada no início do procedimento e fixada placa ponte de $2.8 \mathrm{~mm}$ (fixada no navicular e $2^{\circ}$ metatarso). A sequência da técnica cirúrgica está demonstrada na - Figura 3. O tecido ósseo necrótico e desvitalizado retirado do cuneiforme foi enviado para exame anatomopatológico, que confirmou o diagnóstico de osteonecrose.

O paciente permaneceu 8 semanas sem apoio, seguidos de 3 meses de reabilitação fisioterápica. Naquela ocasião, já apresentava melhora clínica importante. Durante o acompanhamento, os exames de imagem de controle mostraram incorporação do enxerto ósseo no osso cuneiforme intermédio (-Figura 4).
Após a total consolidação, procedemos ao segundo tempo do tratamento, que ocorreu 6 meses após a primeira cirurgia. Nesta segunda intervenção, foi realizada a retirada do material de síntese e correção da deformidade cavo varo sutil do pé. Através do acesso dorsal prévio, retirou-se a placa e foi visualizado um cuneiforme intermédio biologicamente viável, estável, e com incorporação do enxerto ósseo ( - Figura 5). Após a retirada da placa e liberação articular, realizamos o tratamento do pé cavo varo sutil com osteotomia de extensão do $1^{\circ}$ metatarso, liberação da fáscia plantar e alongamento do gastrocnêmio medial.

O paciente apresentou ótima evolução, realizou reabilitação física com retorno às atividades esportivas sem intercorrências. Após 3 meses da $2^{\mathrm{a}}$ cirurgia o paciente já praticava corridas leves e exercícios sem impacto na academia.

Na última avaliação, ocorrida 16 meses após a $2^{\mathrm{a}}$ cirurgia, o paciente relatou que realizava corrida e atividades de impacto sem limitações ou queixas álgicas. Ele classificou o resultado como excelente e relatou que realizaria novamente o procedimento.
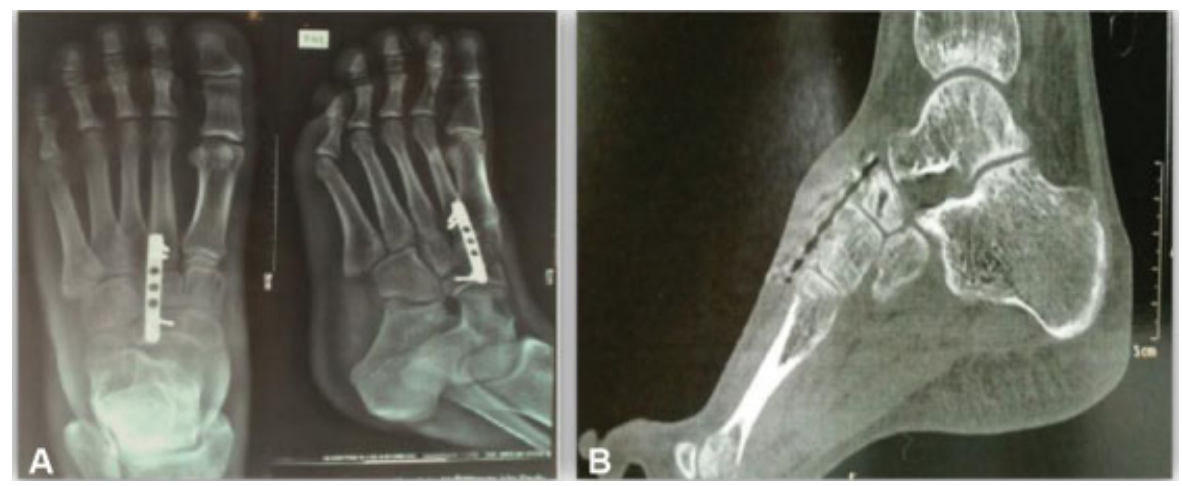

Fig. 4 Exames de imagem realizados após seis meses da cirurgia. Evidenciamos incorporação do enxerto ósseo. (A) Radiografia. (B) Tomografia computadorizada. 


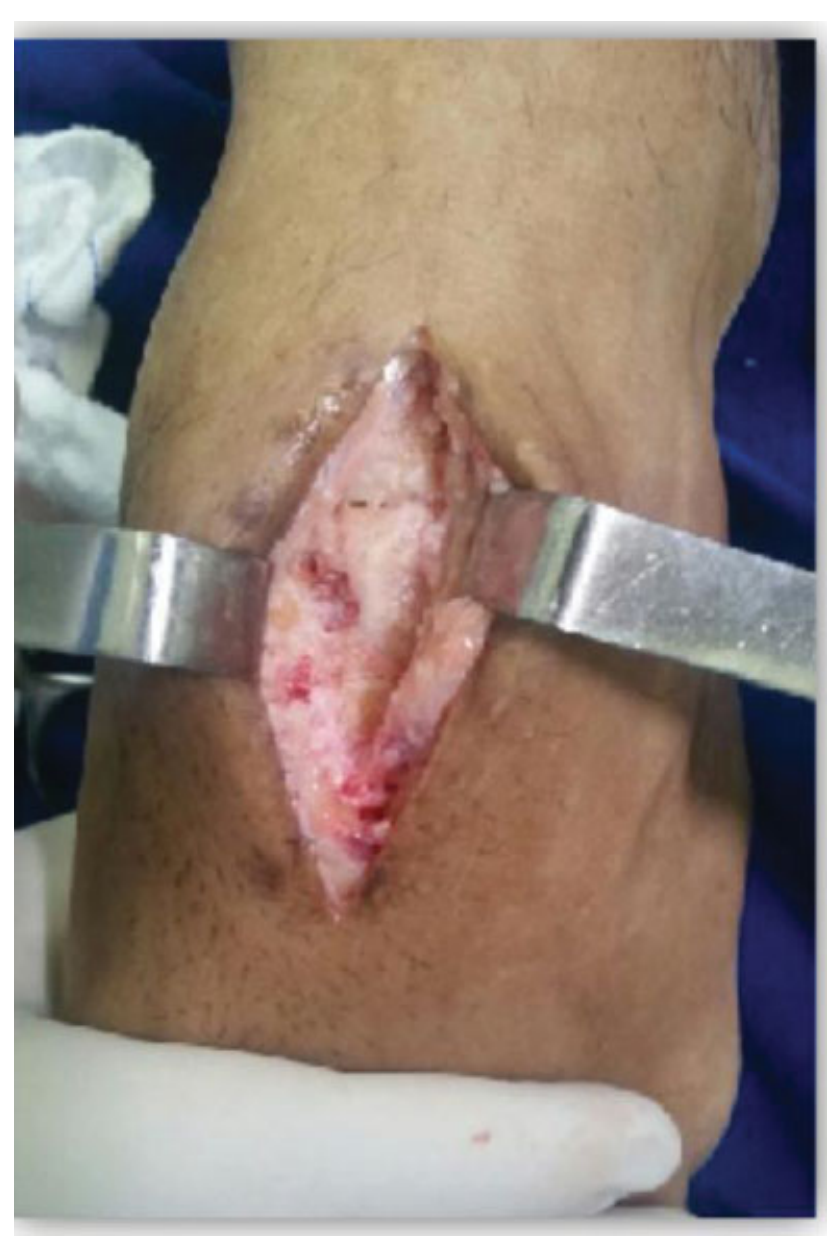

Fig. 5 Vista dorsal do mediopé mostrando o aspecto intra-operatório do osso cuneiforme intermédio após a retirada da placa. Fonte: Arquivo pessoal do autor.

\section{Discussão}

Existem diferentes formas de abordagem para o tratamento da osteonecrose, porém a maioria das publicações aborda o tratamento da necrose da cabeça do fêmur. No pé e na articulação do tornozelo, a literatura faz referência com maior ênfase ao acometimento do tálus e do navicular. ${ }^{1,2}$ Após revisão da literatura, foram encontrados apenas cinco artigos relatando casos de osteonecrose do osso cuneiforme intermédio em pacientes pediátricos tratados conservadoramente. ${ }^{3-5}$ Não foi encontrado relato desta patologia na literatura nacional.

O tratamento da necrose avascular dos ossos do tarso ainda é controverso e não possui protocolos específicos; portanto, é possível afirmar que a primeira escolha deve ser conservadora com repouso, retirada de carga, órteses, e adequação física. ${ }^{1,2}$ Em vários relatos dessa patologia em outros sítios anatômicos os pacientes apresentaram melhora clínica com essa conduta. ${ }^{2-4}$

Em relação ao tratamento cirúrgico, nos quadros mais precoces sem degeneração articular estão indicadas cirurgias que preservam a articulação. Esta abordagem pode ser alcançada por algumas técnicas cirúrgicas, como a descom- pressão óssea intramedular, realizada isoladamente ou associada ao uso de enxerto ósseo que pode ser vascularizado ou não. $O$ enxerto ósseo vascularizado do cuboide é uma cirurgia descrita para tratar casos inicias de osteonecrose do tálus com mínimo colapso subcondral. ${ }^{6}$ Alguns autores mostraram ótimos resultados de pacientes com osteonecrose do tálus tratados com esta técnica. ${ }^{6}$ Em contrapartida, Chew et al. ${ }^{7}$ mostraram uma osteonecrose do osso cuneiforme medial tratada somente através de microperfurações com bons resultados clínico e radiográfico. Considerando a raridade e a heterogeneidade desta patologia, não existem trabalhos comparativos que contemplam uma técnica superior para o tratamento da osteonecrose nas fases iniciais. ${ }^{6} 0$ que de fato podemos afirmar é que em casos avançados com degeneração articular, a artrodese é o procedimento ideal que permite a manutenção do comprimento e da arquitetura óssea. ${ }^{3}$ Apesar do paciente do estudo não apresentar sinais degenerativos, tratava-se de uma osteonecrose avançada, pois durante a abordagem cirúrgica foi observado acometimento ósseo de toda a extensão do osso cuneiforme intermédio associado a comprometimento subcondral, e, devido a isso, os autores optaram pelo uso de enxerto ósseo não vascularizado em grande quantidade para preenchimento completo do canal medular.

Outra variante do tratamento é a maneira de fixar o enxerto. McLeod et al. ${ }^{2}$ mostraram um caso de osteonecrose da tíbia na qual realizou-se abertura da lesão com janela óssea anterior, curetagem, e retirada dos tecidos necróticos, seguidos de preenchimento com enxerto ósseo e fixação com placa para estabilizar o enxerto. Essa abordagem foi utilizada no presente estudo ao realizar uma fixação com placa ponte, que foi fixada ao navicular e ao segundo metatarso. Os autores acreditam que a estabilização do enxerto é essencial para obtenção do sucesso no tratamento. A fixação com placa, além de estabilizar o enxerto, contribui para diminuir o estresse mecânico ao dissipar a energia naquela região, favorecendo o processo de reintegração óssea.

A osteonecrose pode ser secundária a fraturas por estresse causadas por microtraumas de repetição e fatores intrínsecos representados por deformidades e desequilíbrios musculares que alteram a biomecânica e a distribuição da carga nos membros inferiores. Em seu estudo, Li et al. ${ }^{9}$ observaram a relação do alinhamento do retropé com a osteonecrose do navicular. Trataram 14 pés com necrose do navicular somente com o realinhamento do retropé, feito através da osteotomia valgizante do calcâneo, sem abordar diretamente o navicular. Observaram ótimos resultados clínicos e radiográficos, reforçando a relação do alinhamento e da sobrecarga biomecânica que ocorre no mediopé através de deformidades do membro inferior. ${ }^{9}$ De acordo com BuiMansfield et al., ${ }^{10}$ os cuneiformes, devido à sua localização anatômica, estão susceptíveis a forças de compressão, que podem ser exacerbadas por alterações do eixo mecânico, desequilíbrios musculares e, especificamente, por patologias da fáscia plantar. $O$ paciente deste estudo apresentava como fator intrínseco um pé cavo varo sutil associado a um encurtamento do gastrocnêmio, e, devido a isto, após retirar a placa, foi efetuada a correção do pé cavo varo sutil obtida 
com a osteotomia de extensão do primeiro metatarso, fasciotomia plantar e alongamento do gastrocnêmio. Os autores enfatizam que a restauração da biomecânica deve ser uma etapa complementar do tratamento, pois corrige um fator intrínseco relacionado à gênese da patologia em questão, melhorando os resultados funcionais e reduzindo a chance de recorrência. Em relação à correção da deformidade, por se tratar de um pé cavo varo sutil, flexível, proveniente de um equinismo aumentado do primeiro raio, que corrigia no primeiro tempo dos blocos de Coleman, o tratamento ocorreu através de uma osteotomia de extensão do primeiro metatarso associado aos procedimentos de partes moles já descritos.

Os autores apresentam um caso raro de osteonecrose do osso cuneiforme intermédio em um paciente adulto tratado cirurgicamente com descompressão e enxertia óssea fixada temporariamente com placa ponte, seguida pelo realinhamento do eixo mecânico, com ótimo resultado clínico.

\section{Suporte Financeiro}

Não houve suporte financeiro de fontes públicas, comerciais, ou sem fins lucrativos.

Conflito de Interesses

Os autores declaram não haver conflito de interesses.

\section{Referências}

1 DiGiovanni CW, Patel A, Calfee R, Nickisch F. Osteonecrosis in the foot. J Am Acad Orthop Surg 2007;15(04):208-217

2 McLeod JM, Ng A, Kruse DL, Stone PA. Nontraumatic Osteonecrosis of the Distal Tibia: A Case Presentation and Review of the Literature. J Foot Ankle Surg 2017;56(01):158-166

3 García-Mata S. Avascular necrosis of the intermediate cuneiform bone in a child: a very rare cause of limp in a child. A variant of the normality? J Pediatr Orthop B 2013;22(03):255-258

4 Kose O, Demiralp B, Oto M, Sehirlioglu A. An unusual cause of foot pain in a child: osteochondrosis of the intermediate cuneiform. J Foot Ankle Surg 2009;48(04):474-476

5 Watmough PJ, Tselentakis G, Day JB. Avascular necrosis of the intermediate cuneiform bone. J Pediatr Orthop B 2003;12(06):402-405

6 Cody EA, Nunley JA. Vascularized Pedicle Graft for Talar Osteonecrosis. Foot Ankle Clin 2019;24(01):121-129

7 Chew JT, Low CK, Mitra AK. A case report on bilateral avascular necrosis of the medical cuneiforms. Ann Acad Med Singapore 1995;24(03):453-454

8 Gangji V, De Maertelaer V, Hauzeur JP. Autologous bone marrow cell implantation in the treatment of non-traumatic osteonecrosis of the femoral head: Five year follow-up of a prospective controlled study. Bone 2011;49(05):1005-1009

9 Li SY, Myerson MS, Monteagudo M, Maceira E. Efficacy of Calcaneus Osteotomy for Treatment of Symptomatic Müller-Weiss Disease. Foot Ankle Int 2017;38(03):261-269

10 Bui-Mansfield LT, Thomas WR. Magnetic resonance imaging of stress injury of the cuneiform bones in patients with plantar fasciitis. J Comput Assist Tomogr 2009;33(04):593-596 